\title{
CORONARY DISEASE: A PATHOLOGICAL STUDY
}

BY

\author{
P. J. D. SNOW, A. MORGAN JONES, AND K. S. DABER \\ From the Departments of Cardiology and Pathology, University of Manchester
}

Received February 9, 1955

Since the diagnosis and management of coronary disease depend largely upon premises derived from pathological (and clinico-pathological) studies, the methods used in these types of investigation obviously require careful scrutiny. The focal point of such work is the detailed examination of the heart at necropsy, and the more accurate the methods used for this purpose, the greater the number of lesions revealed in the coronary arteries and myocardium. In previous work the introduction of new technical methods for studying either arterial or myocardial lesions has led to concentration upon the type of lesion that the new technique demonstrated, often to the comparative neglect of other lesions. Thus the detailed methods of examining the myocardium employed by Kossmann and de la Chappelle (1938), Lowe (1941) and Myers et al. (1947) were not combined with equally satisfactory methods of examining the coronary arteries, and the very careful study of the coronary arteries made by Blumgart et al. (1940) does not appear to have been combined with an equally comprehensive study of the myocardium. It therefore seemed worthwhile to reinvestigate the relationship between coronary artery occlusion and myocardial infarction by the best available techniques for the post-mortem study of both arterial and myocardial lesions.

\section{Methods}

The method used to examine the coronary arteries was that devised by Schlesinger (1938). In addition, however, a detailed myocardial examination was made, with a comprehensive histological examination of all suspected arterial and myocardial lesions. Only an outline of the technique is given here for a detailed description will be published elsewhere. At autopsy the heart was removed entire and the coronary arteries cannulated under saline. The heart was then suspended in saline at $44^{\circ} \mathrm{C}$. and the arteries perfused with normal saline till the washings returned clear. The radiopaque injection medium introduced by Schlesinger was then injected at a pressure of $140 \mathrm{~mm}$. $\mathrm{Hg}$. The heart was cooled in iced saline and unrolled so as to lie in one plane. A radiograph of the specimen was obtained by means of which all occlusions and points of narrowing can be recognized. Using the radiograph as a guide, the arteries were sectioned at 3-4 mm. intervals to confirm and extend the radiographic findings by naked eye and microscopic examination. Since the injection medium was red in the right coronary artery and blue in the left, the colour of the medium distal to an occlusion indicated the source of collateral channels. Serial sections were then taken through the whole heart at intervals of $0.5 \mathrm{~cm}$. The myocardium was scrutinized and all suspicious portions removed for microscopic examination. If no naked eye abnormality was found, sections were routinely taken from the anterior, lateral, and posterior walls of the left ventricle and the posterior wall of the right, these being the sites at which infarcts are most likely to occur.

In assessing the number of infarcts, confluent or superimposed areas of infarction were regarded as separate incidents if the age of each portion was demonstrably different from that of contiguous areas. In practice this differentiation presents little difficulty. It is, however, necessary to emphasize that there are few areas of infarction in which the whole mass of muscle is necrosed: indeed if this did occur ventricular aneurysm or rupture would be the usual sequel of myocardial infarction. 


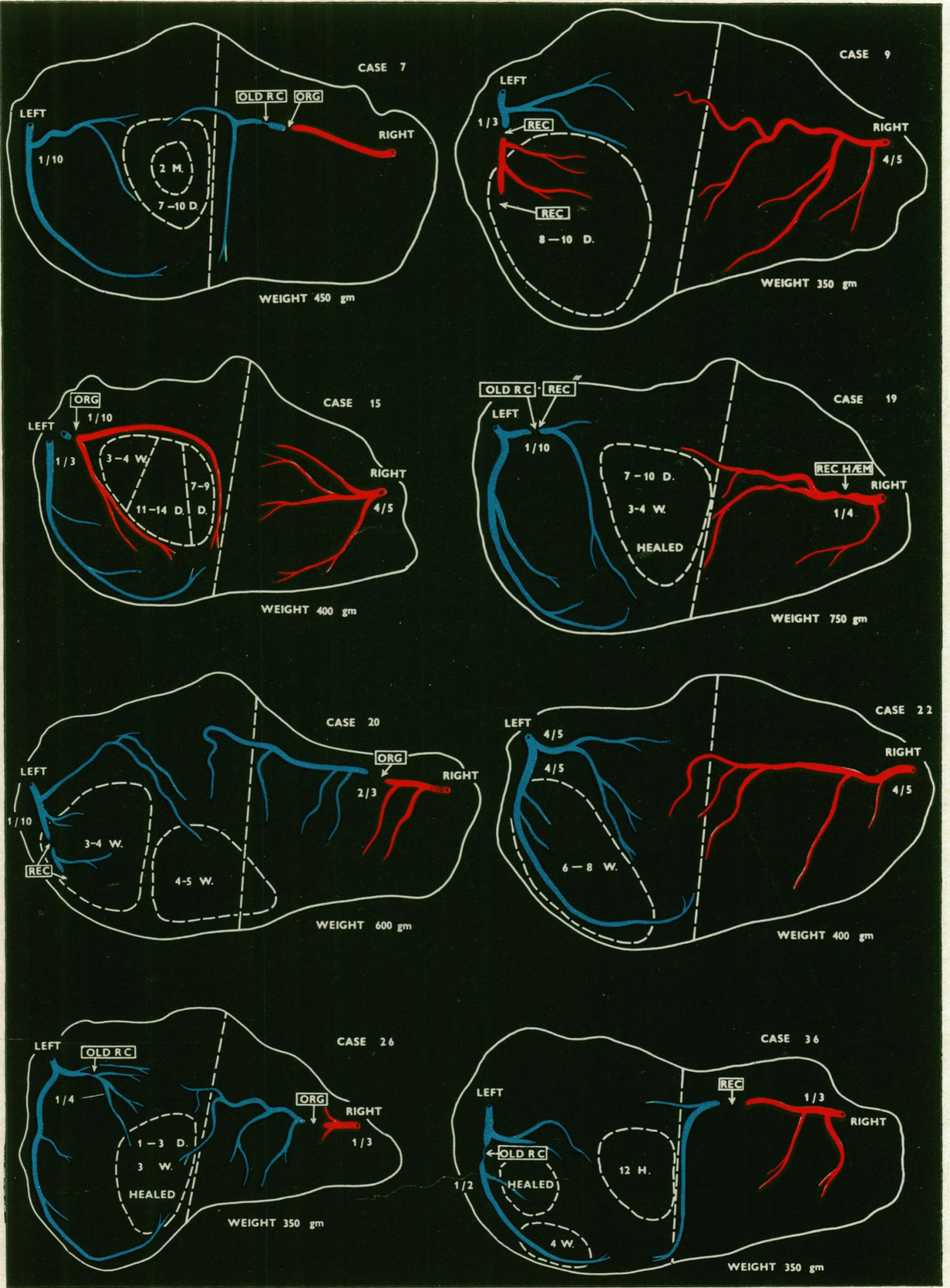


In fact, throughout an infarcted area there remains a network of surviving muscle, which forms a living framework holding the islands of necrotic tissue together. These appearances are, of course, to be distinguished from the histological differences between the periphery and the centre of the same infarct due to more rapid healing at the periphery. Superimposition of successive incidents of infarction may therefore occur in the same area of myocardium. This concept is essential not only for the understanding of the pathology of infarction but also to appreciate the electrocardiographic features associated with extension of an infarct.

The possibility that some healed infarcts may have been due to aggregation of small areas of ischæmic fibrosis was considered, but in this series the same reticular pattern was found in old as in recent lesions, relatively large areas of fibrous replacement alternating with viable muscle. In ischæmic fibrosis, on the other hand, the replacement of muscle takes place fibre by fibre, and the more homogeneous appearance is readily distinguishable from that of infarction.

The ages of infarcts were assessed according to the criteria of Mallory et al. (1935). In our cases comparison of this assessment with the actual date of infarction, when this was clinically established, indicates that this method permits ageing within a day or two during the first week, within 4 or 5 days up to 3 weeks, and within 2-3 weeks up to 3 months. Unfortunately it was found impossible to assess the age of occlusions with a corresponding degree of accuracy for there seems to be considerable variation in the rate of organization.

Revascularized atheromatous plaques are to be distinguished from recanalized occlusions, which show the following features.

(1) Injection medium in the revascularized channels is in direct continuity with the medium in the artery proximal and distal to the arterial narrowing. This can be confirmed both histologically and radiographically.

(2) If more than one revascularized channel is present, all channels run parallel to the main arterial lumen.

(3) Cellular connective tissue is present in the wall of the revascularized channel.

For ease of assessment, all the pathological data were recorded in diagrammatic form on tracings of the injected heart (Fig. 1).

FIG. 1

Key: REC, recent occlusion; ORG, organizing occlusion; OLD R.C., old recanalized occlusion; D, days; W, weeks; $M$, months; H, hours; REC. HAEM., recent intimal hæmorrhage. The broken line divides the right and left ventricles.

Case 7.-An example of a recanalized occlusion transmitting a functionally significant blood supply. A more recent occlusion just proximal to it has caused a further infarct.

Case 9.-An example of occlusion without infarction. The more recent distal occlusion in the anterior descending branch of the left coronary artery has occurred within an already infarcted area.

Case 15.-An example of three confluent infarcts of different ages resulting from one occlusion. The presence of an extensive collateral circulation did not prevent extension of infarction. Note that the circumflex and anterior descending arteries arise by separate orifices.

Case 19.-An example in which there is some difficulty in interpretation. An old occlusion of the left circumflex branch led to a healed posterior infarct. This occlusion recanalized and subsequently reoccluded. The two more recent infarcts were probably due to the recent re-occlusion of the left circumflex branch and the hæmorrhagic narrowing of the right coronary respectively. It is possible, however, that both these infarcts could have arisen from either recent coronary obstruction. The left circumflex artery beyond the occlusion has been revascularized from the anterior descending branch.

Case 20.-A second example of an occlusion without a corresponding infarct. Both occlusions lie in the anterior descending branch of the left coronary artery within an infarcted area.

Case 22.-The only example in this series of an infarct occurring without any corresponding occlusion or significant narrowing of the coronary arteries.

Case 26.-The old recanalized occlusion of the circumflex branch of the left coronary artery is associated with a healed infarct at the apex of the left ventricle. A more recent occlusion of the right coronary artery has led to a 3-weekold infarct, which subsequently extended (1-3 days before death) into islands of surviving myocardium (superimposed extension).

Case 36.- An example of a single occlusion in the anterior descending branch of the left coronary artery causing two infarcts, separated by an area of uninfarcted myocardium. There is also a recent occlusion of the right coronary artery associated with a very recent infarct. It will be noted that there is some collateral vascularization from the left coronary artery. Collateral channels between the two coronary arteries may have been opened up following the old occlusion of the left coronary artery which subsequently recanalized. 


\section{MATERIAL}

In order to make a comprehensive study of coronary disease, we have not confined our attention to cases with clinical manifestations, but have included cases of sudden death and patients in the appropriate age groups without clinical coronary disease who have died from other causes. The present paper is, however, limited to the first 25 cases of clinical coronary disease. Although the data presented here are purely pathological, it is intended to correlate them with the detailed clinical observations (which are also available in these cases) and this will form the subject of a separate paper.

\section{RESULTS}

In 25 cases, 41 main vessel* occlusions and 59 infarcts were found.

Occlusions. Of the 41 occlusions, 17 were old and, rather surprisingly, all had recanalized; 14 showed no signs of organization and were classified as recent while 10 were of intermediate age and incompletely organized. In the anterior descending branch of the left coronary artery 23 occlusions occurred, 4 in the circumflex branch, and 14 in the right coronary artery.

Infarcts. Of the 59 infarcts, 18 were healed ( 3 months or more old) and 41 were sufficiently recent to permit histological estimation of their age. Two infarcts were found in the right ventricle in one heart, but all other infarcts were in the left ventricle or septum.

Occlusion without Infarction. In only 4 instances had occlusion of a main coronary artery failed to cause myocardial infarction. In each case the explanation was obvious, for the occlusion was the most recent of two or more in the same vessel, lay distal or immediately proximal to the earlier one and the area supplied by the vessel was already infarcted (Fig. 1, Cases 9 and 20).

Infarction without Occlusion. Only 4 areas of infarction were found without complete occlusions of the corresponding main coronary artery. In one case (Fig. 1, Case 22) a large infarct was present with only trivial narrowing of the arteries and without any clinical suggestion of major extra-coronary causes of generalized myocardial ischæmia. Another recent infarct (Fig. 1, Case 19) was associated with recent acute narrowing of a main coronary artery due to intimal hæmorrhage, and two other healed infarcts were associated with severe long-standing atheromatous narrowing of the corresponding arteries.

Multiple Infarcts due to a Single Occlusion. If we exclude these isolated incidents of infarction or occlusion, there remain 37 occlusions which had given rise to 55 infarcts. The additional 18 infarcts occurred in 13 cases in areas which could have been rendered ischæmic by existing occlusions. In 10 of these 13 cases a single occlusion had led to an infarct that subsequently extended on one or more occasions without further occlusion, so that there were contiguous or superimposed areas of infarction of demonstrably different ages (Fig. 1, Cases 15 and 26). In 2 of these 10 cases, the later infarct was adjacent to and confluent with the first, whereas in the other 8 cases the first and subsequent infarcts were superimposed, the islands of tissue surviving from the first infarct being affected by the later lesion. Either of these processes can reasonably be described as "extension" of an infarct, but we have been able to find little reference to them in previously published work on this subject. In the remaining 3 cases the infarcts were separated by an area of uninfarcted myocardium (Fig. 1, Case 36). In 9 cases the interval between the first and subsequent infarcts (due to a single occlusion) was known; in no case did this interval exceed eight weeks and in 7 cases it was less than three weeks. In our cases extension of infarction was not due to extension of the causative occlusion or to new occlusion, but to necrosis of already ischæmic muscle, a fact of some importance in considering the function of anticoagulant therapy.

It is notable that of the 18 instances of multiple infarction due to a single occlusion 16 were recent incidents. This is inevitable because the incidents of infarction are recognizable as separate events principally by the differing ages of the various areas, and when the infarcted area becomes fully healed this distinction is lost. It is impossible to say whether a completely healed area of infarction represents one or several incidents.

Revascularization of Infarcts. In radiographic injection studies of the coronary arteries areas of recent infarction may show up because there are few filled vessels in them (Fig. 2). As time passes the infarcted area becomes revascularized (Fig. 2) and is then indistinguishable on the radiograph though, of course, easily recognizable by naked eye and histological examination. Of the 59 infarcts in our series, 45 could be classified into "filled" or " unfilled" groups. These observations are correlated with the ages of the

* The term " main vessel" is used for the three principal coronary vessels-the right coronary artery and the anterior descending and circumflex branches of the left coronary artery. 


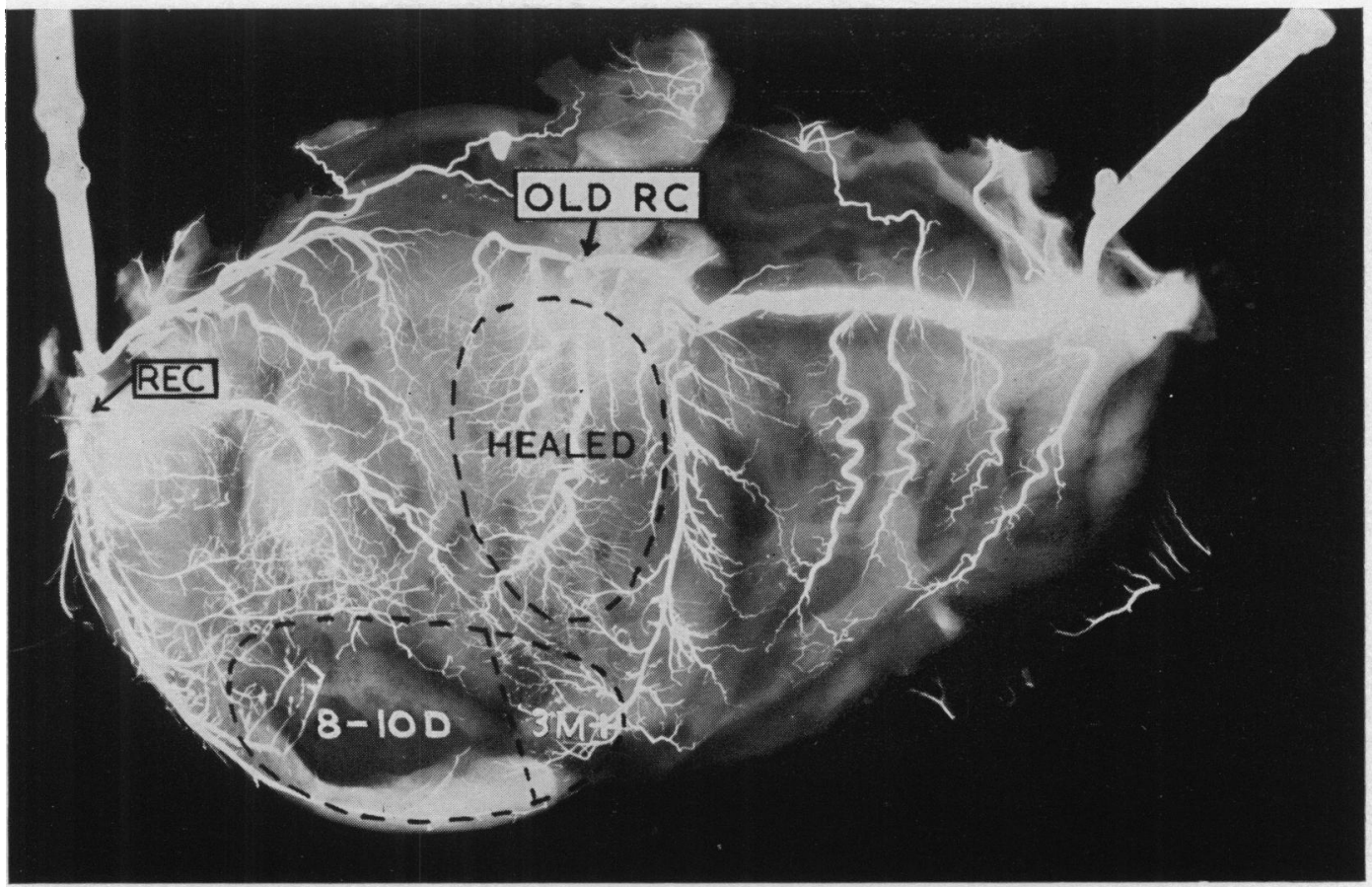

FIG. 2.-Radiograph of coronary arteries injected by Schlesinger's method. Three infarcts are present histologically. The healed and 3-months-old infarcts are well filled with injection mass, whereas the 8-10-day-old infarct is almost unfilled.

infarcts in Fig. 3. With the exception of three infarcts less than a week old, there is no evidence of arterial filling until the third week. Thereafter the proportion of infarcts that fill with injection medium increases until by the eighth week all infarcts are filled. It is concluded that revascularization of an infarcted area takes 3 to 8 weeks, the time probably depending upon the adequacy of the available collateral channels and the size of the infarct.

Three recent infarcts, aged 5-6 hours, 24-28 hours, and 5-6 days respectively, showed apparently normal filling (Fig. 3). Filling up to 24 hours may be explained by the fact that the arterioles in an infarct probably do not necrose as rapidly as the myocardium but this could scarcely explain the filling of an infarct 5-6 days old. The presence of injection medium in the infarct, distal to the occlusion, implies the presence of a collateral circulation which would aid survival of the vessels.

Collateral vascularization is not the only possible blood supply to an area of myocardium once supplied by a vessel that has become occluded. On the contrary, a fairly high proportion of old occlusions recanalize and this was true of all 17 old occlusions in our series. In 3 of these cases, further occlusions developed either in the recanalized channel or immediately adjacent to it, and in each case a further infarct resulted. A recanalized vessel can, therefore, provide a blood supply that is functionally significant.

\section{Discussion}

In 1940 Blumgart, Schlesinger, and Davis undertook the first major study of coronary disease by the improved technique of coronary injection introduced by Schlesinger in 1938. In addition to their convincing demonstration of the frequency and extent of the collateral circulation in coronary disease, they put forward a new and important concept when they claimed that occlusion of a main coronary artery occurred very frequently in man without any corresponding myocardial infarction. This fitted in with their appraisal of the importance of the collateral circulation and has had a considerable influence upon the way in which both pathologists and clinicians have since thought about 
the sequence of events in the heart in coronary disease. It became easy for the pathologist to accept conspicuous coronary occlusion in the absence of demonstrated infarction, and when a prolonged attack of cardiac pain occurred without the associated changes of infarction in the electrocardiogram, the clinician was ready to attribute it to coronary occlusion without infarction. This concept naturally modified our clinical views upon the management of such cases.

When we began this study of coronary disease we expected to be able to confirm the views of Blumgart and his colleagues and anticipated that we would obtain figures for the incidence of infarction after occlusion similar to theirs in which, in 30 cases, 53 main vessel occlusions gave rise to only 16 myocardial infarcts. To our surprise, however, our own results have been strikingly different for in 25 cases, 37 of 41 main vessel occlusions led to at least one corresponding infarct in each instance. Further, in each of the four instances of occlusion without infarction, the occlusion was one of two in the same vessel, and the area supplied by that vessel was infarcted, so it is not surprising that the second occlusion failed to cause a second infarct. In conformity with traditional views, we are therefore obliged to conclude that when obstruction of a main coronary artery occurs in the human subject, it is almost inevitable that myocardial infarction will follow.

It seems important to discover the reasons for this major discrepancy between the results of two rather similar studies. Naturally we wondered whether there was any possibility that we had underestimated the frequency of occlusions in our cases, but on comparing our figures with those of 
Blumgart and his colleagues we found that the incidence of occlusions was almost exactly the same in the two series (Table I). This table also shows that the discrepancy lies not in the frequency of occlusion, but in the frequency of infarction. Our technique for the recognition of myocardial

TABLE I

COMPARATIVE INCIDENCE OF CORONARY OCCLUSION AND FREQUENCY OF INFARCTION

\begin{tabular}{|c|c|c|}
\hline & $\begin{array}{l}\text { Blumgart, Schlesinger, } \\
\text { and Davis (1940) }\end{array}$ & $\begin{array}{l}\text { Present } \\
\text { series }\end{array}$ \\
\hline 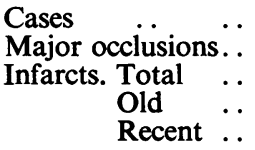 & $\begin{array}{l}30 \\
53(1 \cdot 8 \text { per case }) \\
16(0 \cdot 5 \text { per case }) \\
10 \\
6\end{array}$ & $\begin{array}{l}25 \\
41 \text { ( } 1.6 \text { per case }) \\
59(2.4 \text { per case }) \\
18 \\
41\end{array}$ \\
\hline
\end{tabular}

infarction consisted of a full search of the whole myocardium by the serial slice technique with histological examination of every suspected area, whether naked-eye changes were present or not. So far as we are able to ascertain from their published data no such examination was made in the cases of Blumgart and his colleagues. We are compelled to conclude that the discrepancy may be due, at least in part, to their failure to identify all infarcts, and we believe that this may have misled them in their estimate of the incidence of occlusion without infarction.

In considering the likelihood of occlusion without infarction in the human subject, it must be borne in mind that there is some evidence that major coronary occlusion can be induced in animals without causing infarction. Gregg et al. (1939) found that in the dog gradual occlusion of a main coronary artery in successive stages was often not accompanied by myocardial infarction, although sudden occlusion of a vessel of comparable size was followed by infarction. Gradual coronary occlusion cannot, of course, be recognized with certainty in human pathological studies, but in our cases there were 9 occlusions in vessels previously narrowed at that point by atheroma so that they appeared to be one-tenth or less of their original lumen. It seems reasonable to regard such occlusion as merely the final stage of a gradual narrowing, and in these circumstances there had been time for the development of a collateral circulation. None the less, in all these cases the final occlusion of the narrowed artery led to an infarct. So even comparatively gradual occlusion of a main coronary artery in man leads to infarction as a rule, and in this respect the human subject does not follow the pattern of animal experiments. The most obvious explanation for this difference is that in experimental animals the remaining unoccluded vessels are healthy whereas in the human subject they are often grossly narrowed, or already occluded, so that it is more difficult for an adequate collateral circulation to develop.

When we try to appraise the place of the collateral circulation in preserving the myocardium when coronary occlusion occurs, our results lead us to a conclusion rather different from that of Blumgart et al. (1940), for we find no evidence that the collateral circulation is capable of preventing infarction when a major coronary artery is occluded. Its function might lie in limiting the area of infarction, or in the prevention of subsequent extension of the infarct into adjacent ischæmic zones. We have therefore been interested in trying to study the frequency with which the area of infarction extends and in comparing the time during which extension may occur with the time necessary for the full development of the collateral circulation.

In 10 of our 25 cases a single occlusion resulted in an infarct that subsequently extended on one or more occasions without further occlusion. In 9 instances, the ages of the first and subsequent infarcts were accurately known and in no case did the interval between them exceed 8 weeks, and in 7 cases it was less than 3 weeks. The rate of revascularization of the peripheral ischæmic zone might be expected to determine the period during which extension of an infarct is liable to occur. 
In our series the available evidence suggests that revascularization becomes complete between the third and the eighth weeks (Fig. 3).

Thus it seems that the liability for extension of an infarct passes away as the collateral circulation develops. Our views upon the function of the coronary collateral circulation in man are, therefore, that it probably cannot develop soon enough to prevent infarction when a major coronary artery is occluded, but that it may well serve to limit the area of infarction, and that its major function is the restoration of an adequate circulation to the surviving but ischæmic peripheral zone, so abolishing by degrees the tendency for extension of necrosis into adjacent ischæmic areas.

\section{SUMMARY}

Twenty-five hearts from patients with clinical coronary disease have been subjected to detailed post-mortem examination, including coronary injection and comprehensive histological examination of the myocardium. The concept of the prevention of infarction by the development of collaterals concomitantly with gradual narrowing was not borne out. On the contrary, almost all occlusions (even in greatly narrowed arteries) gave rise to infarcts, and although collaterals developed they did not prevent infarction. Recanalized occlusions appear to be capable of transmitting a functionally significant blood supply for if they re-occlude new infarction follows.

Not only do major occlusions almost invariably cause infarction but in addition a single occlusion frequently gives rise to more than one infarct. In fact, extension of an infarct into adjacent ischæmic zones is a commonly observed pathological event, and this occurs without further coronary occlusion. This tendency for an infarct to extend exists for a period of 3 to 8 weeks after the original infarct, and this period corresponds closely with the time required for the full development of collateral vascularization of the ischæmic area. It is reasonable to conclude that the collateral circulation, when fully developed, plays an important part in preventing the extension of infarction by revascularizing adjacent ischæmic areas of myocardium.

We are indebted to Professor Crighton Bramwell for his interest in this work, to Professor A. C. P. Campbell for his advice on the pathological aspects and to Dr. D. A. K. Black for help in presentation of the results.

\section{REFERENCES}

Blumgart, H. L., Schlesinger, M. J., and Davis, D. (1940). Amer. Heart J., 19, 1.

Gregg, D. E., Thornton, J. J., and Mautz, F. R. (1939). Amer. J. Physiol., 127, 161.

Kossmann, C. E., and de la Chapelle, C. E. (1938). Amer. Heart J., 15, 700.

Lowe, T. E. (1941). Amer. Heart J., 21, 326.

Mallory, G. K., White, P. D., and Salcedo-Salgar, J. (1939). Amer. Heart J., 18, 647.

Myers, G. B., Klein, H. A., Stofer, B. E., and Hiratzka, T. (1947). Amer. Heart J., 34, 785.

Schlesinger, M. J. (1938). Amer. Heart J., 15, 528. 